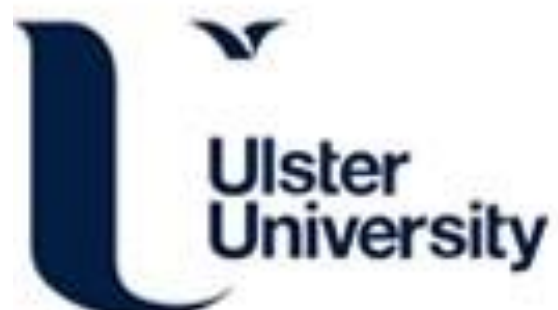

\section{Augmenting Motor Imagery Learning for Brain-Computer Interfacing Using Electrical Stimulation as Feedback}

Bhattacharyya, S., Clerc, M., \& Hayashibe, M. (2019). Augmenting Motor Imagery Learning for Brain-Computer Interfacing Using Electrical Stimulation as Feedback. IEEE Transactions on Medical Robotics and Bionics, 1(4), 247-255. https://doi.org/10.1109/TMRB.2019.2949854

Link to publication record in Ulster University Research Portal

Published in:

IEEE Transactions on Medical Robotics and Bionics

Publication Status:

Published (in print/issue): 30/11/2019

DOI:

10.1109/TMRB.2019.2949854

\section{Document Version}

Author Accepted version

\section{General rights}

Copyright for the publications made accessible via Ulster University's Research Portal is retained by the author(s) and / or other copyright owners and it is a condition of accessing these publications that users recognise and abide by the legal requirements associated with these rights.

\section{Take down policy}

The Research Portal is Ulster University's institutional repository that provides access to Ulster's research outputs. Every effort has been made to ensure that content in the Research Portal does not infringe any person's rights, or applicable UK laws. If you discover content in the Research Portal that you believe breaches copyright or violates any law, please contact pure-support@ulster.ac.uk. 


\title{
Augmenting Motor Imagery Learning for Brain-Computer Interfacing using Electrical Stimulation as Feedback
}

\author{
Saugat Bhattacharyya, Maureen Clerc and Mitsuhiro Hayashibe
}

\begin{abstract}
Brain-computer Interfaces (BCI) and Functional electrical stimulation (FES) contribute significantly to induce cortical learning and to elicit peripheral neuronal activation processes and thus, are highly effective to promote motor recovery. This study aims at understanding the effect of FES as a neural feedback and its influence on the learning process for motor imagery tasks while comparing its performance with a classical visual feedback protocol. The participants were randomly separated into two groups: one group was provided with visual feedback (VIS) while the other received electrical stimulation (FES) as feedback. Both groups performed various motor imagery tasks while feedback was provided in form of a bidirectional bar for VIS group and targeted electrical stimulation on the upper and lower limbs for FES group. The results shown in this paper suggest that the FES based feedback is more intuitive to the participants, hence, the superior results as compared to the visual feedback. The results suggest that the convergence of $\mathrm{BCI}$ with FES modality could improve the learning of the patients both in terms of accuracy and speed and provide a practical solution to the BCI learning process in rehabilitation.
\end{abstract}

Index Terms-Brain-Computer Interfacing, Common Spatial Patterns, Functional Electrical Stimulation, Motor Imagery, Feedback.

\section{INTRODUCTION}

Brain-computer Interfaces (BCI) measures the neural activity of the brain to create a direct communication channel to peripheral devices in form of robots, prosthesis, wheelchair or a computer controlled by the user [1], [2]. Thus, it opens up a new frontier in rehabilitation that would allow the direct control of devices by patients with neural disorders, such as stroke, amyotrophic lateral sclerosis, spinal injury and physical disability [3], [4]. Intentions of the user are generated from the brain in form of electrical impulses or changes in the cerebral blood flow which can be captured by numerous recording modalities, like Electroencephalography, Magnetoencephalography or Functional Magnetic Resonance Imaging. Electroencephalography (EEG) is the most widely used recording technique in the BCI domain because it is inexpensive, portable, easily available and has high temporal resolution [2], [5].

The data acquired from the EEG are used to generate the control signal for the BCI in real time but the performance

Saugat Bhattacharyya is with BCI-NE lab, University of Essex, Colchester CO4 3SQ, UK. e-mail: saugatbhattacharyya@live.com

Maureen Clerc is with Université Côte d'Azur and Inria Sophia AntipolisMéditerranée. e-mail: maureen.clerc@inria.fr

Mitsuhiro Hayashibe is with Department of Robotics and Department of Biomedical Engineering, Graduate School of Engineering, Tohoku University, Sendai, Japan.e-mail: mitsuhiro.hayashibe@ inria.fr of the BCI relies on the users' ability to modulate their brain signals at will. Thus, users participating in BCI experiments undergo an initial training to develop the skill of voluntary brain modulation for a specific cognitive task. One such example is the modulation of rhythmic brain activity in the sensorimotor areas by motor imagery, where the user induces a desynchronization and synchronization (ERD/ERS) in the brain rhythms due to imagining or planning the execution of movement of a certain limb. To provide to the user an indication of his/her voluntary brain modulation, a feedback in form of visual [6], [7], auditory [8] or (vibro-)tactile [9], [10] medium can be provided during training. Feedback in the form of a visual medium is used by most BCI researchers [7] but it is a matter of debate about which feedback modality is most beneficial to the user. There may also be cases when the visual system may be compromised due to a disease or by the nature of the system that the user wishes to control and hence, a different form of feedback must be provided by the system. A study by [7] evaluated the performance of auditory, vibrotactile and visual feedback where the results showed a high variability among individuals. They reached a conclusion that the feedback modality for BCI systems must be personalized for the user. In this paper, we have employed Functional Electrical Stimulation (FES) [11],[12] as a form of feedback in BCI and verified its feasibility as a natural sensory feedback compared to the visual feedback.

FES is often applied during rehabilitation to directly engage muscles located in the impaired section of the body by reconstructing certain daily life skills through direct electrical stimulation. Previous studies have reported the ability of FES to elicit recovery of skills such as standing up, grasping, cycling and walking by re-training the users on these tasks, but most FES based rehabilitative systems do not employ the cortical activity of the patient [13], [14], [15], [16].

One can employ Functional Electrical Stimulation (FES) targeting specific muscle groups as a feedback modality in $\mathrm{BCI}$ research. An existing hypothesis on augmented movement therapy by FES states that recovery occurs mostly due to cortical plasticity and partly due to peripheral mechanisms [17]. This has been confirmed on motor learning tasks involving transcranial magnetic stimulation [18] and functional magnetic resonance imaging [19]. Thus, it is quite natural to combine FES rehabilitation with BCI systems, where the FES can activate the sensory channel to provide a maximal inflow of information into the brain and the BCI would provide an efferent outflow of motor commands from the brain 
to the targeted region in the body (for example, the upper limb) to close the motor loop [20] and this could lead to an improvement of learning for the targeted group.

Studies in [21],[22] have explored the potential of implementing BCI in functional recovery using FES by directly linking the cortical response of the user to muscular response. To date, most BCI-FES systems are used to activate the FES stimulation device through motor imagery commands generated by the BCI. The first study to report restoration of hand grasp was Pfurtscheller et al. in [23] where they combined BCI with FES transmitted via surface electrode. The effects of FES on ERD/ERS patterns during reconstruction of the motor activity was studied by Müller et al. in [24]. EEG/MEG studies undertaken by [22],[25] had also reported the presence of ERD/ERS patterns during an FES induced hand or leg movement tasks. Zhao et al. in [26] demonstrated the feasibility of BCI combined with upper extremity FES to restore functions for patients suffering from tetraplegia. Pistohl et al. in [27] reported the improvement of angular accuracy of movements when the participants were provided with additional artificial proprioceptive feedback rather than using visual feedback but this did not increase the overall accuracy determined from the average distance between the cursor and the target.

The study of effect of FES on the motor cortex and its influence on motor imagery learning is an open area of research. It is interesting to study whether FES has any positive influence as feedback in learning movement-related tasks and, in a more broader sense, for BCI based rehabilitation. Our previous study [28] on FES in the form of a neuro-feedback in BCI has suggested a positive influence of FES during motor learning on the subjects. A steady increase in the classification performance during FES-induced feedback as compared to visual feedback was reported. On incorporating electrical stimulation as neuro-feedback, the subjects in this experiment were reported to be more focused in performing the tasks, especially for the longer sessions.

The primary aim of this paper is to augment the performance of the BCI classifier through FES as a neural feedback and improve the learning process for motor imagery tasks. To meet our goal, electrical stimulation was provided as feedback during two separate but consecutive experimental conditions: when the participants are in a relaxed state (not performing any task) followed by when the participants are performing some active motor imagery task. The former relaxed session is assumed to be a baseline for the later active sessions. The novelty of this paper is the improvement observed in the learning process through electrical stimulation by removing the sensory reaction generated in the brain during stimulation. We also compare the learning during FES and visual feedback. Replacing visual feedback by electrical stimulation could improve the focus of the participants in performing the tasks and augment the performance of the motor task at hand. This in turn would improve the learning of the patients both in terms of accuracy and speed and if successful, it can provide a practical solution to the motor recovery process of the patient.

The rest of the paper is as follows: Section II provides an insight on the experiment protocol adopted for the study of FES

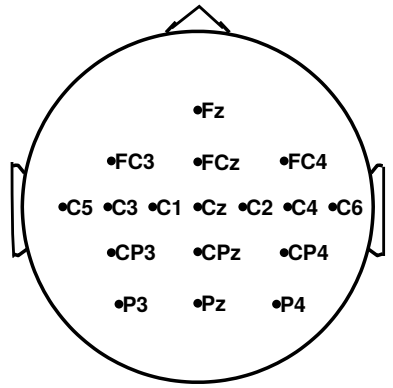

Fig. 1. The locations of the electrodes (or channels) used in the online neurofeedback experiment.

as neuro-feedback during a motor imagery task. Section III illustrates the signal processing techniques adopted to extract relevant features which are to be decoded by the classifier designed specifically for this study. Section IV provides a detailed discussion on the results and their significance. A discussion is included in Section $\mathrm{V}$ followed by the concluding remarks in Section VI.

\section{EXPERIMENT DESCRIPTION}

\section{A. Data Acquisition}

The EEG signals were recorded from 17 channels covering the fronto-central, central, centro-parietal and parietal regions (Fz, FC3, FCz, FC4, C5, C3, C1, Cz, C2, C4, C6, CP3, CPz, $\mathrm{CP} 4, \mathrm{P} 3, \mathrm{Pz}, \mathrm{P} 4)$ (Fig. 1) arranged on the basis of the standard 10-20 system using a TMSI Refa8 amplifier with a sampling rate of $256 \mathrm{~Hz}$.

Surface electrical stimulation is provided to the anterior compartment of the upper-limbs and the triceps surae muscle group for the lower-limbs to induce wrist flexion and foot plantar flexion, corresponding to the mental task to perform. We have used two rectangular electrodes of size $5 \times 9 \mathrm{~cm}$ to relay the stimulation for each limb. For the forearms, the electrodes were placed on the anterior compartment of the forearm for wrist flexion direction. One electrode was placed approximately $3 \mathrm{~cm}$ distal from wrist joint, and the other was placed $1 \mathrm{~cm}$ distal from elbow joint for arm. For stimulation of the triceps surae muscle group to induce foot plantar flexion, one electrode is approximately placed $4 \mathrm{~cm}$ distal from knee joint, and the other one is placed $14 \mathrm{~cm}$ distal from knee joint. The stimulus is delivered by a computer-controlled stimulator (ProStim, MXM, France) with pulse width (PW) modulation [29] $(\mathrm{PW} \max =400 \mu \mathrm{s})$ at a constant amplitude and frequency $(20 \mathrm{~Hz})$. Each stimulation sequence consists of a trapezoidal envelope train of $\mathrm{PW}(0.4 \mathrm{~s}$ ramp-up, $1.2 \mathrm{~s}$ plateau, $0.4 \mathrm{~s}$ ramp-down).

OpenVIBE software [30] was used to record the signal, display the cues, pre-process, analyze and classify the EEG, and to send the commands to the stimulator through MATLAB software during the online run of the experiment. The offline analysis of the EEG is done in MATLAB.

\section{B. Participants}

For this study 16 participants (13 male and 3 female) were recruited with a mean age of 28 years and standard deviation 
of 9 years. 14 of the 16 participants had no prior experience with motor imagery BCI experiments and 2 participants had taken part in a single motor imagery experiment before this current experiment. The experiments took place in INRIA Sophia Antipolis. The electrical stimulation provided to the FES group was kept at a maximum of $25 \mathrm{~mA}$ and before the onset of the experiment, the participants were asked to tune the intensity of the stimulation to a comfortable level of their choosing and this intensity was used for the whole duration of the experiment. The participants performed the experiment in isolation while sitting in a comfortable chair placed in front of a display monitor placed at eye level. Prior to the experiments, the subjects are informed about the purpose of the experiment and the tasks they have to perform. After the experiment, the participants were asked to provide a subjective assessment, in a scale of 1-10, of 'how focussed they were while performing the tasks'. All participants signed an informed consent form before taking part in the study, which was approved by institutional ethical committee.

\section{Task Description}

The participants performed the following cued motor imagery tasks: left hand, right hand, left foot and right foot. In this experiment, the participants were randomly placed in two groups: one group was provided with only visual feedback (VIS) and the other group received only FES feedback during the motor imagery tasks. One of the two participant with prior experience with BCI was placed in the FES group and the other was placed in the VIS group. In this way each group was exclusively trained with only one type of feedback.

The group with FES feedback performed two different sets of experiments: 1) the participant performed the motor imagery tasks while receiving electrical stimulation as feedback, which we call FES-Active (ACT), and 2) the participants performed no motor imagery task and received the electrical stimulation as a cue, which we call FES-Passive (PAS). A session provides instructions to the participant through a sequence of visual cues to execute one of the four motor tasks and each visual cue is associated to a 'trial'. Each participant from the FES group first underwent 1 PAS session followed by 3 ACT sessions in a single experiment run, while each participant from the VIS group underwent 3 sessions. Each session consisted of 40 trials of each motor imagery task. Prior to the feedback session, each participant underwent a training session of 160 trials.

\section{Design of the Visual Cue}

The visual cue (or instruction) is designed similar to our previous work in [28] and a schematic time representation of the visual cue is shown in Fig. 2. Each session starts with a resting period of 20 seconds followed by motor imagery trials. Each trial begins with a fixation cross displayed on screen for 1 second followed by a 1 second instruction of the motor task to be performed in form of arrows. First, a left or right arrow is displayed on screen for 0.5 second to indicate left or right motor imagery, respectively followed by up or down arrow of 0.5 second which corresponds to hand or foot

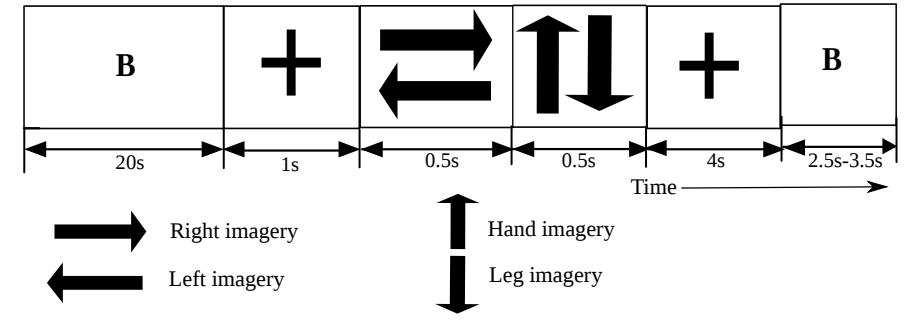

Fig. 2. Schematic of the sequence of visual cues displayed to the participants during the online neuro-feedback experiment.

imagery, respectively. Following the instructions, the feedback is generated for 4 seconds. The subject commences his/her task on the onset of this cue and the feedback received is the motor imagery output of the subject. For FES sessions, only electrical stimulation is provided to the subjects as feedback while for VIS sessions the subjects receive only visual feedback. Lastly, a blank screen is displayed for $2.5 \mathrm{~s}-3.5 \mathrm{~s}$ for the participants to relax and also avoid overlapping of task related EEG between consecutive trials.

\section{Methodology}

A motor imagery (MI) task is characterized by an occurrence of event-related desynchronisation (ERD), resulting in a decrease in sensorimotor rhythms prominently in the mu band $(8-12 \mathrm{~Hz})$ and central beta band (16-24 Hz) [31]. After termination of the MI task, an event-related synchronisation (ERS) occurs most notably as an increase in power in the beta band (13-35 Hz) [32]. Thus, it can be assumed that each MI task has a characteristic signature in these frequency bands. The decoder is trained to identify these characteristic signatures and provide an output for an unknown occurrence. This section describes the pre-processing, feature extraction and classification techniques adopted in this study to generate the output required to display (for VIS group)/generate (for FES) the online feedback and for further offline analysis presented in this study.

\section{A. Pre-processing}

The raw EEG signal is first filtered using a notch filter, to remove the $50 \mathrm{~Hz}$ line noise. Then the signal was filtered using a 4th order Butterworth band-pass filter at [8,24] $\mathrm{Hz}$ [33], [34] to detect and study the ERD. After filtering the signal, the continuous EEG were segmented to lengths of 3 seconds into epochs, starting from 1 second before the onset of feedback period of each motor imagery task.

\section{B. Decoding the Motor Imagery EEG}

After extracting the relevant EEG epoch, Common Spatial Patterns (CSP) [35] were computed to project the multichannel EEG data into low-dimensional spatial subspace with a projection matrix, capable of maximizing the variance ratio of the two class signal matrices. It is based on the simultaneous diagonalization of the covariance matrices of both classes. 
Let us define a single trial EEG as $\mathbf{X} \epsilon \mathbf{R}^{C h \times T}$, where $C h$ is the number of channels and $T$ is the number of samples per channel. Thus, by using the CSP algorithm one can project $\mathbf{X}$ to a spatially filtered counterpart $\mathbf{Z}$, by

$$
\mathbf{Z}=\mathbf{W X}
$$

where, rows of the projection matrix $\mathbf{W}$ are the spatial filters and the columns of $\mathbf{W}^{-1}$ represent the CSP. The computation of the CSP is explained thoroughly in [35],[36]. The first and last columns of the spatial patterns correspond to the largest variance of one task and the smallest variance of the other. In our study, we have selected two pairs (the first and the last two) of optimal spatial filters. Then, we had calculated the variance $\left(\mathrm{VAR}_{p}\right)$ of the 4 spatially filtered time series for an epoch $T$.

Lastly, the variances are normalized and log-transformed to generate 4 feature vectors.

$$
f_{p}=\log \left(\frac{\mathrm{VAR}_{p}}{\sum_{p=1}^{4} \operatorname{VAR}_{p}}\right) .
$$

The feature vectors are used as inputs to the Linear Discriminant Analysis (LDA) classifier [37], which has a low computational cost and is simple to use. It separates the data representing the different classes based on a boundary function, known as the hyperplane. The hyperplane is a projection that maximizes the distance between two class means, while minimizing the inter-class variance [37]. Thus, the class of an observation depends on which the side of the hyperplane its feature vector lies. For multi-class classification between four tasks (left hand, right hand, right foot and left foot MI), the classifier first classifies between left and right MI followed by hand and foot MI. This approach was first presented in [28] and adopted in this experiment to generate the commands for feedback control. A schematic of the study is shown in Fig. 3.

\section{Online Generation of Commands for Feedback Control}

The pipeline of the BCI designed to generate the output for the neuro-feedback is similar to the one designed in our previous work [28] and is shown in Fig. 4. The classifier described above, produces output in the form of output labels. For FES sessions, the neuro-feedback is provided to the limb corresponding to the output label generated by the classifier for 4 seconds. PW modulation depended on the classifier output: hyperplane distance with a fixed amplitude and fixed time length (4s). For VIS sessions, the feedback is provided to the subject in the form of a blue bar, where the predicted label determines the direction of the bar with respect to the fixation cross (the bar appears to the right of the fixation cross for correct classification output and it appears to the left of the fixation cross for incorrect ones). The length of the bar changes with the hyperplane distance obtained from the classifier. For example, if the classifier produces an output of right foot instead of the true right hand label, then the bar would appear on the left side. Whereas if the classifier yields an output of right hand, then the bar would appear on the right

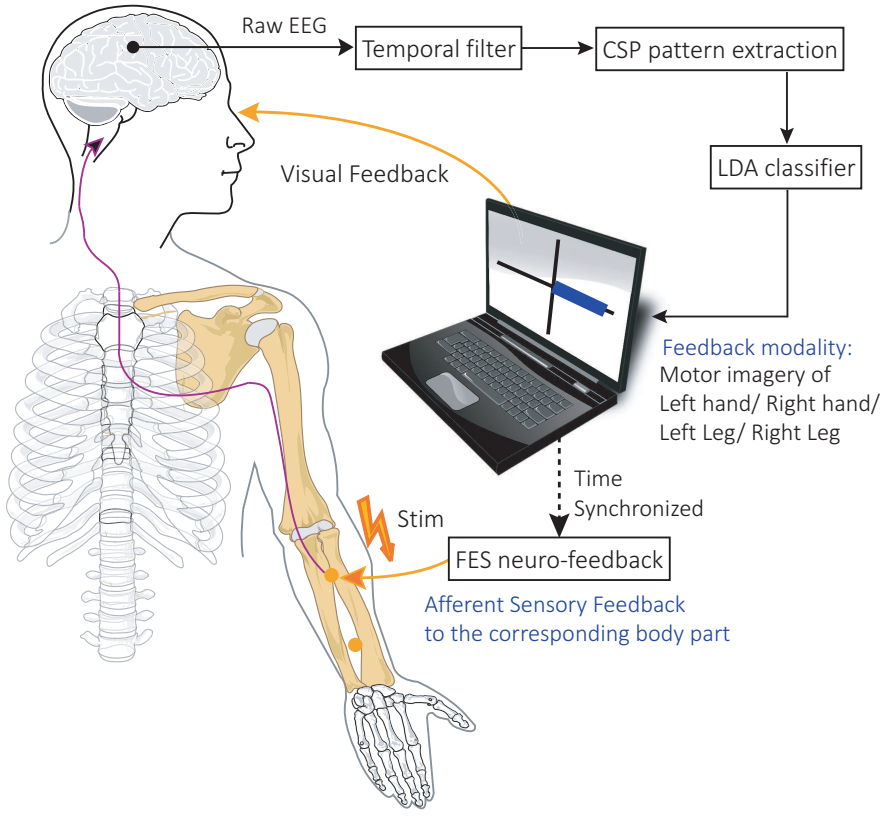

Fig. 3. A schematic of the neuro-feedback experiment adopted for this study. Participants within the FES group are provided with electrical stimulation on their limbs as a feedback while participants within the VIS group are provided with visual feedback.

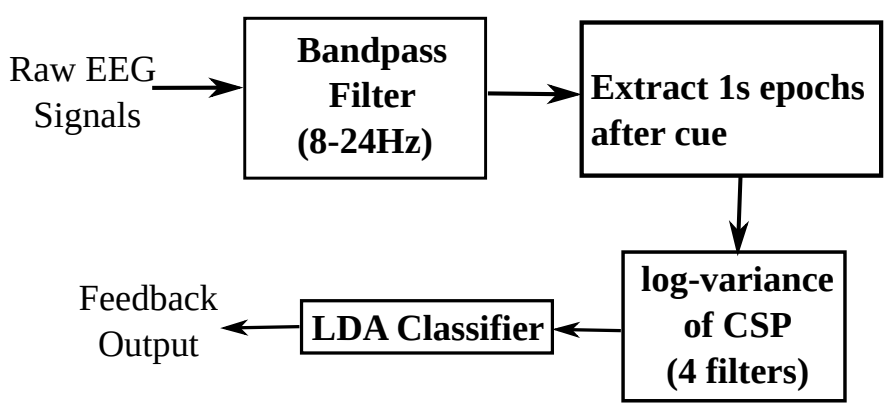

Fig. 4. Pipeline of the BCI to generate the neuro-feedback from the incoming EEG.

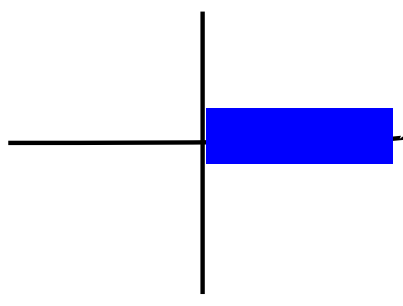

(a)

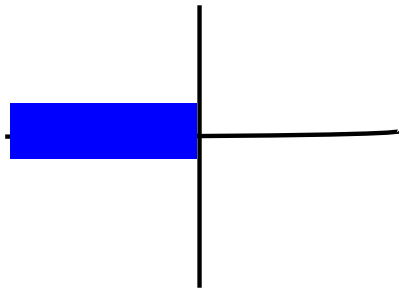

(b)
Fig. 5. A simulation of the feedback as visualized by subjects performing the VIS sessions. (a) shows the feedback for correct classification and (b) illustrates the feedback for incorrect classification. The length of the bar is determined by the hyperplane distance of the output label.

side. An illustration of the feedback displayed to the subjects is shown in Fig. 5.

\section{Offline relabelling and Analysis of EEG Data}

Our previous research [28] had studied the effects of FES while learning a motor imagery task. The study showed a 
significant improvement in performance accuracy when the user received an electrical stimulation as feedback in place of the visual one. The current study further seeks to improve the performance of the BCI with FES as a medium of feedback and compare its performance to VIS feedback. The occurrence of learning in both the FES and visual feedback are analysed from the EEG obtained from the three experimental conditions: ACT, PAS and VIS. To further improve the analysis of data during ACT conditions, we have removed the average bandpower of the EEG obtained during the PAS session from the bandpower of the EEG obtained during the ACT sessions (named as ACT-PAS). We have employed the spectrogram method [34] to calculate the band power at a frequency range between $8 \mathrm{~Hz}$ to $12 \mathrm{~Hz}$ and sliding time-window of $100 \mathrm{~ms}$ for EEG epochs of $3 \mathrm{~s}$, starting from $1 \mathrm{~s}$ before the onset of feedback period. The average band power for PAS session was calculated across all trials of the same motor imagery task for an epoch length of 3s. The reason behind this step is that during PAS sessions, the subjects were involved in no motor related activity. Thus, this condition contains the information of EEG when the brain reacts passively to the electrical stimulation. Therefore, we can consider the PAS as a baseline to the ACT with ACT containing both the passive reaction and the motor imagery information by receiving the sensory feedback including proprioception. By removing the PAS component from its ACT counterpart, we can filter out the sensory reaction in the brain originating from the electrical stimulation and specifically consider the activities that correspond to augmentation in motor imagery learning thanks to the sensory feedback.

Furthermore, to simplify the offline analysis, we have combined the data of right and left foot imageries into a single class called 'foot imagery'. In this paper, we have three motor imagery classes: left hand, right hand and foot. The ACT and VIS consists of 40 trials/session $\times 3$ sessions $=120$ trials of left and right hand classes and 80 trials/session $\times 3$ sessions $=$ 240 trials of foot classes. The PAS consists of 40 trials/session $\times 1$ sessions $=40$ trials of left and right hand classes and 80 trials/session $\times 1$ sessions $=80$ trials of foot classes. To maintain parity with the online approach, we have kept the pre-processing parameters similar to the one described in section III-A. We have designed a unique two-level decoding technique to re-train and re-tune the CSP and LDA parameters to the new class labels. Each layer of the classifier was trained with data collected during the training session prior to the feedback sessions (see Section II-C). In the first level (FL), we designed three CSP sub-filters and LDA sub-classifiers to decode the following events: left-hand (class 1) vs \{right-hand and foot $\}$ (class 0) (Sub-classifier FL1), right-hand (class 1) vs \{left-hand and foot (class 0) (Sub-classifier FL2) and foot (class 1) vs $\{$ left-hand and right-hand $\}$ (class 0 ) (Sub-classifier FL3). If one of the sub-classifier yields an output of class 1 and the other two classifiers produce an output of class 0 , then the event for which the sub-classifier produced the class 1 output is the final result of that epoch. For example, as shown in Table I, if sub-classifier FL1 produces an output of 1 and the other two sub-classifiers produces 0 , then left-hand imagery is the final output as the left-hand event was assigned as class 1
TABLE I

ClassifIER DESIGN

\begin{tabular}{cccccc}
\hline \multicolumn{7}{c}{ Level 1 decoding } \\
\hline Sub-classifier & Left & Right & Foot & Status & 2nd level \\
\hline FL1 & 1 & 0 & 0 & Left output & - \\
FL2 & 0 & 1 & 0 & Right output & - \\
FL3 & 0 & 0 & 1 & Foot output & - \\
\hline SL1 & 1 & 1 & 0 & Tie & Left vs Right \\
SL2 & 1 & 0 & 1 & Tie & Left vs Foot \\
SL3 & 0 & 1 & 1 & Tie & Right vs Foot \\
\hline
\end{tabular}

in sub-classifier FL1. In the case when two FL sub-classifiers produce an output of 1 , then the features are re-classified in the second level (SL) to determine the final output of the system. For example, if both sub-classifier FL1 and sub-classifier FL3 produced an output of 1, then the features of left hand and foot events (sub-classifier SL2 is activated) are re-classified among themselves to determine the final output (which may be left hand or foot). Table I presents the two-level decoding technique.

To detect if learning occurred during the feedback period of each successive trial, we have taken the absolute value of hyperplane distance only in the correct direction [37] (the distance of a point from the decision boundary) as a performance metric during three conditions: ACT, PAS, ACT-PAS and VIS. One can assume that the greater the hyperplane distance is, the greater is the confidence that the classifier produced a correct decision. Hence, in this study, we have taken the winning hyperplane distance obtained at the end of first or second level classification as the final hyperplane distance of the classifier. Taking an example from Table I, if sub-classifier FL1 produced an output of 1 and FL2 and FL3 produced 0, then the average of the hyperplane distance for class 1 is designated as the final hyperplane distance. In case the second level of the classifier is activated, then the hyperplane distance of the winning label is nominated as the final hyperplane distance of the classifier. The final hyperplane distance is then used to study the effects of learning across successive trials. To study the effects of learning across sessions or the overall performance of each feedback condition, we have used the classification accuracy as an evaluation index along with the hyperplane distance.

\section{RESUlTS}

\section{A. Signal Representation}

Fig. 6 represents the grand-averages of the ERD/ERS representation at electrode location $\mathrm{C} 4$ during the onset of the left hand motor imagery tasks for ACT (in blue), PAS (in red), VIS (in green) and ACT-PAS (in black). Except the PAS condition, all the other conditions show a prominent decrease in the power in between $650 \mathrm{~ms}$ to $800 \mathrm{~ms}$ after the onset of stimuli followed by a rise in power (which may indicate the presence of ERS). The dotted lines indicate the p-values of a two-tailed Wilcoxon signed-rank test [38] at each time instance where the ERD of ACT-PAS is compared with ACT (blue dotted), PAS (red dotted) and VIS (green dotted). The black dotted horizontal line represents the 5\% significance level and 


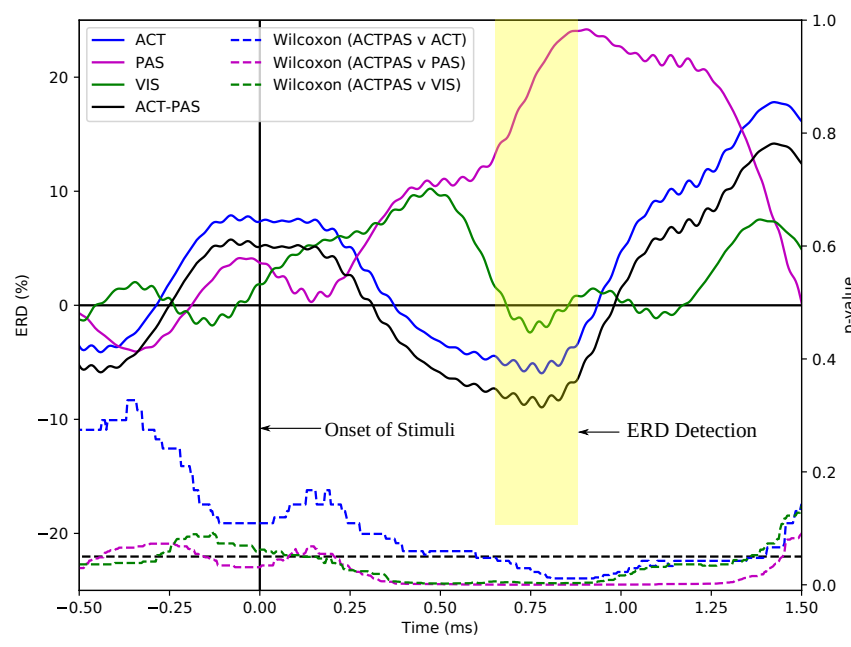

Fig. 6. Grand averages of the ERD at electrode $\mathrm{C} 4$ while performing the left hand motor imagery tasks during ACT (in blue), PAS (in red), VIS (in green) and ACT-PAS conditions (in black) and the corresponding p-values of the two-tailed Wilcoxon signed-rank test at each time instance when ACT-PAS is compared with ACT (in dotted blue), PAS (in dotted red) and VIS (in dotted green). The black dotted horizontal line represents the 5\% significance level. A similar representation was obtained for right hand and foot motor imagery.

any p-values below it suggests significant difference between the two conditions.

The PAS condition suggests that without any motor imagery task being performed by the participants, no conclusive effect of motor activity occurs in the brain and it contains information on the brain sensory reaction purely due to FES feedback. On the other hand, by removing the PAS component from the ACT, we notice a removal of offset of the ERD (see blue dotted line). By looking at the ACT and ACT-PAS representation, we can infer that a prominent ERD with a greater attenuation is present as compared to the VIS condition.

\section{B. Learning Across Successive Trials}

First, we report the learning during ACT, PAS, VIS and ACT-PAS for each motor imagery task using the absolute value of the hyperplane distance of the output label. We took this parameter to study the feedback effect because the larger the hyperplane distance is, the higher the confidence of the classifier is to detect the right output. Fig.7 presents the grand-average of hyperplane distance for 40 successive trials pertaining to a single motor imagery task over all 3 sessions along with their respective p-values using Kruskal-Wallis [39] for each interval of session. This test is a non-parametric method for testing whether two or more independent samples of equal or different sizes originate from the same distribution.

From the figure, it is noted that there is a steady and significant increase ( $p$-value $<0.01$ ) in the hyperplane distance for ACT, VIS and ACT-PAS which suggests that as the participants repeat the task, the participants are more confident in performing the task which is reflected in the EEG features. The ACT conditions have similar learning to VIS conditions for left hand imagery (such behaviour could be because of the nature of VIS feedback where the correct responses were shown by a right bar), poorer to VIS for right hand imagery and better than VIS for foot imagery. Thus, in this comparison, it is difficult to ascertain whether the ACT is better than VIS. However, by removing the baseline component (PAS) from the ACT signals, we can certainly infer that the FES feedback promotes better learning than the standard VIS paradigm. The difference in performance of the three conditions: ACT, ACTPAS and VIS were also found to be significant using Kruskalwallis statistical test for the three motor imagery tasks: left hand motor imagery ( $\mathrm{p}$-value $=0.00001)$, right hand motor imagery $(p$-value $=0.00007)$ and foot motor imagery ( $p$-value $=0.003)$.

\section{Classification Performance of all Participants}

The overall classification accuracy and the mean of the hyperplane distance, obtained from the final correctly classified trial, for each participant after three sessions of ACT, VIS and ACT-PAS are shown in Table II. ACT-PAS performs better in terms of accuracy than the VIS and ACT sessions by $9.14 \%$ and $9.88 \%$, respectively. Six participants in the ACTPAS condition and four participants in the ACT conditions have higher performance accuracy than their VIS counterpart. ACT-PAS also has a bigger hyperplane distance in comparison to ACT and VIS conditions. This clearly indicates that ACTPAS shows better learning and superior accuracy than ACT and VIS conditions.

We have used a two-tailed Wilcoxon signed-rank test [38] to find whether the differences in performance across different sessions are significant or not. It is noted from the p-values in Table II, that ACT-PAS is significantly superior to ACT and VIS conditions, both in terms of accuracy and hyperplane distance while it is inconclusive whether ACT is superior to VIS. Hence, we again conclude that by taking into account the sensory reaction signal component, motor imagery changes in ACT data could be highlighted. Thus, by subtracting the PAS component from the ACT signals, the FES neuro-feedback is significantly better than visual feedback in training a participant to perform motor imagery tasks.

\section{Classifier Performance across Consecutive Intervals}

Next, we have looked into the mean classification accuracy of the participants undergoing the ACT and VIS sessions at different trial intervals and compared it with ACT-PAS. Each participants in the ACT and VIS sessions underwent 480 trials (120 for left hand and right hand imagery task and 240 for foot imagery) of motor imagery. In this analysis, we had divided the 480 trials into 10 consecutive intervals starting from 48 trials and ending at 480 trials and the results for ACT, VIS and ACT-PAS are shown in Fig. 8.

The accuracy obtained during ACT-PAS is shown to have significantly superior performance (Two-tailed Wilcoxon signed-rank test; $\mathrm{p}<0.03$ ) to ACT and VIS. Similar to previous results, there is no significant difference between the accuracies of ACT and VIS conditions and it is again inconclusive whether the ACT condition alone is better than VIS or vice versa. It is also noted that the difference in accuracy between the ACT-PAS and ACT or VIS conditions are maximum at smaller trial sizes and the difference gradually 


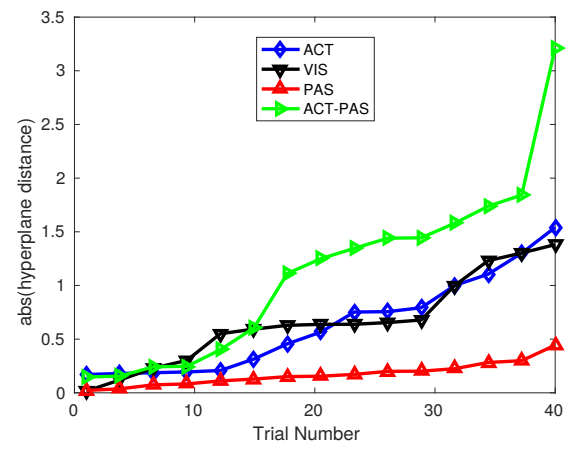

Left hand imagery

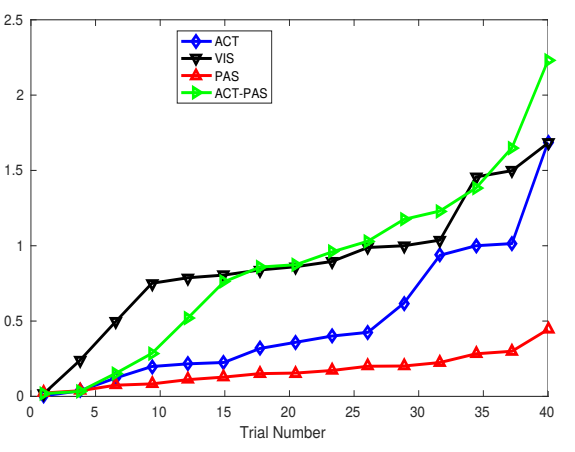

Right hand imagery

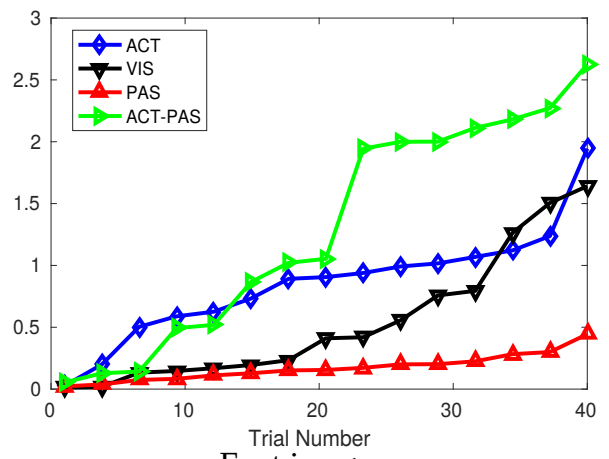

Foot imagery

Fig. 7. The grand-average of hyperplane distance for the three MI imageries over 40 sucessive trials across all participants for the three experimental conditions (FES in blue, FES-PAS in red and VIS in black).

TABLE II

OVERALl PERFormANCE

\begin{tabular}{|c|c|c|c|c||c|c|}
\hline Participant & \multicolumn{3}{|c|}{ Accuracy } & \multicolumn{3}{c|}{ Mean hyperplane distance } \\
\hline & ACT & VIS & ACT-PAS & ACT & VIS & ACT-PAS \\
\hline 1 & 88.89 & 80.36 & 96.87 & 2.5459 & 1.5358 & 3.6095 \\
2 & 96.55 & 91.07 & 97.22 & 4.0306 & 3.2524 & 4.2877 \\
3 & 90.48 & 87.50 & 100.00 & 3.0703 & 2.4901 & 5.2004 \\
4 & 83.33 & 100.00 & 97.22 & 2.4901 & 5.0344 & 4.3053 \\
5 & 72.22 & 72.22 & 96.67 & 1.4317 & 1.3480 & 3.5187 \\
6 & 83.33 & 88.89 & 100.00 & 2.7323 & 3.1698 & 5.0344 \\
7 & 90.48 & 84.84 & 94.12 & 3.4813 & 2.1711 & 4.2299 \\
8 & 90.62 & 96.97 & 92.86 & 3.5112 & 4.6095 & 3.6329 \\
\hline Mean & 86.99 & 87.73 & 96.87 & 2.9117 & 2.9514 & 4.2273 \\
Std & 6.87 & 8.30 & 2.33 & 0.7496 & 1.2561 & 0.5964 \\
\hline Is ACT better than VIS? & $p=0.866$ & $p=\mathbf{0 . 0 1 2}$ \\
Is ACT-PAS better than ACT? & $p=\mathbf{0 . 0 1 2}$ & $p=\mathbf{0 . 0 1 2}$ \\
Is ACT-PAS better than VIS? & $p=\mathbf{0 . 0 3 6}$ & $p=\mathbf{0 . 0 1 2}$ \\
\hline
\end{tabular}

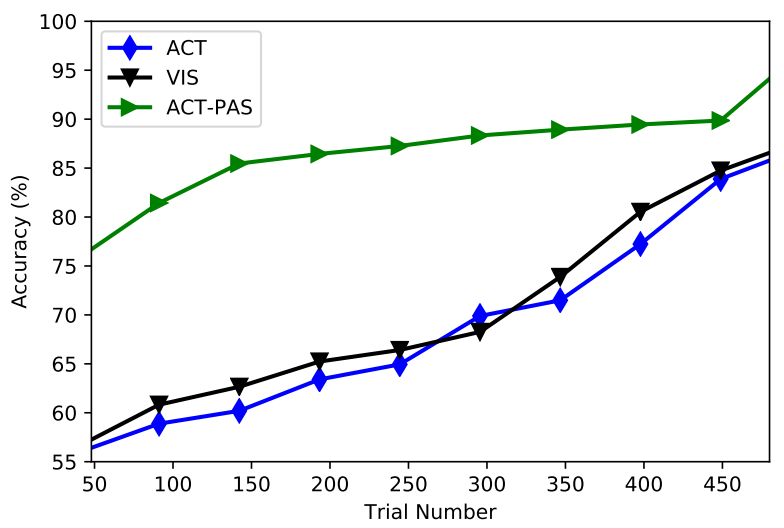

Fig. 8. Mean classification accuracy of 8 participants for ACT (in blue), VIS (in black) and ACT-PAS (in green) conditions from trial 48 to 480 for 10 consecutive trials.

narrows down for the larger trial sizes. Hence, by removing the sensory reaction (PAS) from the motor imagery signals during ACT, we can say that the FES based neuro-feedback is inherently better and requires a much smaller user training time than the visual feedback.

\section{DISCUSSION}

In this paper, we have augmented the performance of motor imagery BCI by applying functional electrical stimulation (FES) as neuro-feedback. The participants that underwent FES training took part in two different sessions: one while performing motor imagery tasks (ACT) and the other without performing any motor imagery task (PAS). The EEG during PAS session comprised only of sensory reaction of brain by having sensory feedback including proprioception through FES. Hence, by removing the sensory reaction, the ACT sessions exclusively contained signals related to motor imagery tasks. The features of these signals in turn, lead to improvement in the BCI performance as well as provide insight on whether the FES has any effect on motor imagery learning.

For this study, we have employed CSP to extract the relevant features and LDA to classify the mental intentions of the participants. Classification was used to compare the performance of the different neuro-feedback conditions (ACT, PAS, ACT-PAS and VIS) across trials, across sessions and overall. We have used the hyperplane distance of the output as an attribute to learning with neuro-feedback by the participants across successive trials in a session. It is assumed that the bigger the hyperplane distance is, the more distinguishable the features are from each other. This suggests that due to the repetitions in the tasks, the brain gradually encodes a signature 
EEG to the motor imagery task which can be more easily decoded by the system.

The results mentioned in Section IV show a significant improvement in performance and learning when the PAS component is removed from the EEG obtained during the ACT conditions. The steep increase in hyperplane distance seen in Fig. 7 across successive correctly classified trials for the three conditions: ACT, ACT-PAS and VIS suggests an improvement in learning among participants to differentiate between the different motor imagery tasks during the run of the experiment. The results also show that the ACT-PAS yields better results and requires shorter training time than the standard visual (VIS) sessions (Table II and Fig. 8). The superior results by ACT-PAS suggest that sensory feedback through FES is more effective in motor imagery learning than the standard visual feedback. Moreover, the average of the subjective assessment (see sectionII-B) of the participants did report a more focused approach towards the task when they were provided with FES feedback than the VIS feedback.

The results seen after incorporating electrical stimulation as feedback in the motor training of participants are positive and provide an incentive to pursue this feedback modality in future BCI research. A sensory feedback including proprioception related to motor control is more natural than visual stimuli which requires user's attention to visual feedback. Even though the experiments in this study were simple and easy for the participants, further fine-tuning is required for future studies. Even though the performance of the BCI improved due to FES feedback, it is still not clear as how the removal of sensory reaction (PAS) led to this improvement. For better understanding of the implications of electrical stimulation on motor imagery learning, experiments with better control conditions are required. Few suggestions provided by the reviewers of this paper include giving stimulation to the contralateral limb while the participant performs a motor imagery task (for example, stimulating the left hand while the subject performs a right hand motor imagery), removal of passive component from VIS tasks by including a "completely" passive condition while resting. Future analysis will include the extension of this methodology to both the lower limbs as opposed to the three limbs used in this study. Also, the experiment may be tested on a more interactive visual medium and a combination of feedback (say, visual and electrical stimulation) for comparison with the FES results in this study.

\section{CONCLUSION}

This work provides conclusive evidence on the enhancement in motor imagery learning due to electrical stimulation as feedback. The results show a significant increase in learning across successive trials and sessions for the same motor imagery task with sensory feedback including proprioception through electrical stimulation. The results further outline the efficacy of incorporating feedback for improvement of the BCI performance, as it tunes the participants to a particular task over the course of the experiment. Fig. 7 shows that the FES and VIS group produces a steady and significant increase in performance. Further, the superior performance by the FES group in Table II validates our claim that FES yields an intuitive and more interactive motor imagery learning. The results suggests that FES feedback would be a suitable alternative to visual feedback in rehabilitative applications. The merger of Brain-computer Interfacing methods (BCI) with Functional electrical stimulation (FES) is highly suitable in the motor recovery process because both modalities contribute to the cortical and peripheral learning process. This in turn should aid patients in reducing the re-learning time.

Further studies on a larger group of subjects are required to validate the effectiveness of electrical stimulation in BCI feedback. Also, alternatives to the feature extraction and classification techniques need to be studied to further improve the decoding performance of the BCI. Positive results from these studies will lead to an improvement in motor imagery based control which would aid in neuroprosthetic control [40].

\section{REFERENCES}

[1] R. P. N. Rao and R. Scherer, "Brain-computer interfacing [in the spotlight],' IEEE Signal Processing Magazine, vol. 27, no. 4, pp. 152 150, July 2010.

[2] J. van Erp, F. Lotte, and M. Tangermann, "Brain-computer interfaces: Beyond medical applications," Computer, vol. 45, no. 4, pp. 26-34, April 2012.

[3] G. Dornhege, Toward Brain-computer Interfacing, ser. A Bradford book. MIT Press, 2007.

[4] L. F. Nicolas-Alonso and J. Gomez-Gil, "Brain computer interfaces, a review," Sensors, vol. 12, no. 2, pp. 1211-1279, 2012. [Online]. Available: http://www.mdpi.com/1424-8220/12/2/1211

[5] J. d. R. Millán, R. Rupp, G. Mueller-Putz, R. MurraySmith, C. Giugliemma, M. Tangermann, C. Vidaurre, F. Cincotti, A. Kubler, R. Leeb, C. Neuper, K. Mueller, and D. Mattia, "Combining brain-computer interfaces and assistive technologies: State-of-the-art and challenges," Frontiers in Neuroscience, vol. 4, p. 161, 2010. [Online]. Available: https://www.frontiersin.org/article/10.3389/fnins.2010.00161

[6] E. Thomas, J. Fruitet, and M. Clerc, "Combining erd and ers features to create a system-paced bci," Journal of Neuroscience Methods, vol. 216, no. 2, pp. 96 - 103, 2013. [Online]. Available: http://www.sciencedirect.com/science/article/pii/S0165027013001416

[7] I. N. Angulo-Sherman and D. Gutiérrez, "Effect of different feedback modalities in the performance of brain-computer interfaces," in 2014 International Conference on Electronics, Communications and Computers (CONIELECOMP), Feb 2014, pp. 14-21.

[8] K. A. McCreadie, D. H. Coyle, and G. Prasad, "Is sensorimotor bci performance influenced differently by mono, stereo, or 3-d auditory feedback?" IEEE Transactions on Neural Systems and Rehabilitation Engineering, vol. 22, no. 3, pp. 431-440, May 2014.

[9] R. Leeb, K. Gwak, D. S. Kim, and J. d. R. Millán, "Freeing the visual channel by exploiting vibrotactile bci feedback," in 2013 35th Annual International Conference of the IEEE Engineering in Medicine and Biology Society (EMBC), July 2013, pp. 3093-3096.

[10] F. Cincotti, L. Kauhanen, F. Aloise, T. Palomaki, N. Caporusso, P. Jylanki, F. Babiloni, G. Vanacker, M. Nuttin, M. G. Marciani, J. del R. Millan, and D. Mattia, "Preliminary experimentation on vibrotactile feedback in the context of mu-rhythm based bci," in 2007 29th Annual International Conference of the IEEE Engineering in Medicine and Biology Society, Aug 2007, pp. 4739-4742.

[11] D. B. Popović, "Advances in functional electrical stimulation (fes)," Journal of Electromyography and Kinesiology, vol. 24, no. 6 , pp. $795-802$, 2014. [Online]. Available: http://www.sciencedirect.com/science/article/pii/S1050641114001953

[12] Q. Zhang, M. Hayashibe, and C. Azevedo-Coste, "Evoked electromyography-based closed-loop torque control in functional electrical stimulation," IEEE Transactions on Biomedical Engineering, vol. 60, no. 8, pp. 2299-2307, Aug 2013.

[13] R. Riener, M. Ferrarin, E. E. Pavan, and C. A. Frigo, "Patient-driven control of fes-supported standing up and sitting down: experimental results," IEEE Transactions on Rehabilitation Engineering, vol. 8, no. 4 pp. 523-529, Dec 2000. 
[14] J. Cauraugh, K. Light, S. Kim, M. Thigpen, and A. Behrman, "Chronic motor dysfunction after stroke," Stroke, vol. 31, no. 6, pp. 1360-1364, 2000. [Online]. Available: http://stroke.ahajournals.org/content/31/6/1360

[15] J. J. Chen, N.-Y. Yu, D.-G. Huang, B.-T. Ann, and G.-C. Chang, "Applying fuzzy logic to control cycling movement induced by functional electrical stimulation," IEEE Transactions on Rehabilitation Engineering, vol. 5, no. 2, pp. 158-169, Jun 1997.

[16] J. Kojović, M. Djurić-Jovičić, S. Došen, M. B. Popović, and D. B. Popović, "Sensor-driven four-channel stimulation of paretic leg: Functional electrical walking therapy," Journal of Neuroscience Methods, vol. 181, no. 1, pp. 100 - 105, 2009. [Online]. Available: http://www.sciencedirect.com/science/article/pii/S0165027009001988

[17] D. B. Popovic and M. B. Popovic, "Hybrid assistive systems for rehabilitation: Lessons learned from functional electrical therapy in hemiplegics," in 2006 International Conference of the IEEE Engineering in Medicine and Biology Society, Aug 2006, pp. 2146-2149.

[18] F. Tyč and A. Boyadjian, "Plasticity of motor cortex induced by coordination and training," Clinical Neurophysiology, vol. 122 , no. 1, pp. $153-162,2011$. [Online]. Available: http://www.sciencedirect.com/science/article/pii/S1388245710004864

[19] J. Liepert, "Evidence-based methods in motor rehabilitation after stroke," Fortschr Neurol Psychiatr, vol. 11, no. 1, pp. 5 - 10, 2010

[20] A. Jackson, J. Mavoori, and E. Fetz, "Long-term motor cortex plasticity induced by an electronic neural implant," Nature, vol. 444, no. 7115, pp. 56-60, 2006.

[21] H. Gollee, I. Volosyak, A. J. McLachlan, K. J. Hunt, and A. Gräser, "An ssvep-based brain-computer interface for the control of functional electrical stimulation," IEEE Transactions on Biomedical Engineering, vol. 57, no. 8, pp. 1847-1855, Aug 2010.

[22] M. Takahashi, M. Gouko, and K. Ito, "Electroencephalogram (eeg) and functional electrical stimulation (fes) system for rehabilitation of stroke patients," in 2008 21st IEEE International Symposium on ComputerBased Medical Systems, June 2008, pp. 53-58.

[23] G. Pfurtscheller, G. R. Muller, J. Pfurtscheller, H. J. Gerner, and R. Rupp, "'thought' - control of functional electrical stimulation to restore hand grasp in a patient with tetraplegia," Neuroscience Letters, vol. 351, no. 1, pp. 33 - 36, 2003. [Online]. Available: http://www.sciencedirect.com/science/article/pii/S0304394003009479

[24] G. Muller, C. Neuper, R., C. Keinrath, H. Gerner, and G. Pfurtscheller, "Event-related beta eeg changes during wrist movements induced by functional electrical stimulation of forearm muscles in man," Neuroscience Letters, vol. 340 , no. 2, pp. $143-147,2003$. [Online]. Available: http://www.sciencedirect.com/science/article/pii/S0304394003000193

[25] S. Salenius, A. Schnitzler, R. Salmelin, V. Jousmäki, and R. Hari, "Modulation of human cortical rolandic rhythms during natural sensorimotor tasks," NeuroImage, vol. 5, no. 3, pp. $221-228$, 1997. [Online]. Available: http://www.sciencedirect.com/science/article/pii/S1053811997902615

[26] Y. Chu, X. Zhao, J. Han, Y. Zhao, and J. Yao, "Ssvep based braincomputer interface controlled functional electrical stimulation system for upper extremity rehabilitation," in 2014 IEEE International Conference on Robotics and Biomimetics (ROBIO 2014), Dec 2014, pp. 2244-2249.

[27] T. Pistohl, D. Joshi, G. Ganesh, A. Jackson, and K. Nazarpour, "Artificial proprioceptive feedback for myoelectric control," IEEE Transactions on Neural Systems and Rehabilitation Engineering, vol. 23, no. 3, pp. 498507, May 2015.

[28] S. Bhattacharyya, M. Clerc, and M. Hayashibe, "A study on the effect of electrical stimulation during motor imagery learning in brain-computer interfacing," in 2016 IEEE International Conference on Systems, Man, and Cybernetics (SMC), Oct 2016, pp. 002 840-002 845.

[29] T. Kesar, L.-W. Chou, and S. A. Binder-Macleod, "Effects of stimulation frequency versus pulse duration modulation on muscle fatigue," Journal of Electromyography and Kinesiology, vol. 18, no. 4, pp. 662 - 671, 2008. [Online]. Available: http://www.sciencedirect.com/science/article/pii/S1050641107000028

[30] Y. Renard, F. Lotte, G. Gibert, M. Congedo, E. Maby, V. Delannoy, O. Bertrand, and A. Lécuyer, "Openvibe: An open-source software platform to design, test, and use brain-computer interfaces in real and virtual environments," Presence, vol. 19, no. 1, pp. 35-53, Feb 2010.

[31] S. Bhattacharyya, A. Konar, and D. N. Tibarewala, "Motor imagery and error related potential induced position control of a robotic arm," IEEE/CAA Journal of Automatica Sinica, vol. 4, no. 4, pp. 639-650, 2017.

[32] C. Neuper and G. Pfurtscheller, "Evidence for distinct beta resonance frequencies in human eeg related to specific sensorimotor cortical areas," Clinical Neurophysiology, vol. 112, no. 11, pp. 2084 - 2097, 2001. [Online]. Available: http://www.sciencedirect.com/science/article/pii/S1388245701006617

[33] P. Herman, G. Prasad, T. M. McGinnity, and D. Coyle, "Comparative analysis of spectral approaches to feature extraction for eeg-based motor imagery classification," IEEE Transactions on Neural Systems and Rehabilitation Engineering, vol. 16, no. 4, pp. 317-326, Aug 2008.

[34] N. Brodu, F. Lotte, and A. Lécuyer, "Comparative study of band-power extraction techniques for motor imagery classification," in 2011 IEEE Symposium on Computational Intelligence, Cognitive Algorithms, Mind, and Brain (CCMB), April 2011, pp. 1-6.

[35] H. Ramoser, J. Muller-Gerking, and G. Pfurtscheller, "Optimal spatial filtering of single trial eeg during imagined hand movement," IEEE Transactions on Rehabilitation Engineering, vol. 8, no. 4, pp. 441-446, Dec 2000.

[36] Y. Wang, S. Gao, and X. Gao, "Common spatial pattern method for channel selelction in motor imagery based brain-computer interface," in 2005 IEEE Engineering in Medicine and Biology 27th Annual Conference, Jan 2005, pp. 5392-5395.

[37] E. Alpaydin, Introduction to Machine Learning (Adaptive Computation and Machine Learning). The MIT Press, 2004.

[38] G. W. Corder and D. I. Foreman, Comparing Two Related Samples: The Wilcoxon Signed Ranks Test. John Wiley \& Sons, Inc., 2009, pp. 38-56. [Online]. Available: http://dx.doi.org/10.1002/9781118165881.ch3

[39] —-, Comparing More than Two Unrelated Samples: The Kruskal-Wallis H-Test. John Wiley \& Sons, Inc., 2009, pp. 99-121. [Online]. Available: http://dx.doi.org/10.1002/9781118165881.ch6

[40] S. Bhattacharyya, S. Shimoda, and M. Hayashibe, "A synergetic brainmachine interfacing paradigm for multi-dof robot control," IEEE Transactions on Systems, Man, and Cybernetics: Systems, vol. 46, no. 7, pp. 957-968, July 2016. 